\title{
Imaginary motion EEG analysis and identification based on AR model
}

\author{
Shi Lijuan, Cui Guixue, Li Zhenxin, Dong Bingchao, Yu Yi \\ Department of Biomedical Engineering \\ Xinxiang Medical University \\ Xinxiang, China \\ shilijuancumt@163.com
}

\begin{abstract}
BCI converted EEG to the corresponding user commands to achieve direct communication and control of the human brain and a computer or other electronic devices. Study on motor imagery EEG feature extraction and classification is an important branch of BCI research. Imaginary movement EEG is defined by the brain to imagine limb movements without actually being generated by the movement. Firstly, the collected C3, C4 EEG channel spectrum and power spectrum were analyzed to find the frequency range that contains significant motion Imaginary feature, whereby the design of suitable band-pass filter for filtering, and uses the independent component analysis (ICA) algorithm de-noising. Doing domain ERD analysis of the filtered EEG to determine the occurrence time of a single test to happen synchronize / sync event, then interception the segment of EEG data from the time domain, and then through auto-regression model, wavelet packet analysis and AR Model power Spectrum analysis of EEG preprocessed feature extraction, and the extracted feature value is input to the two classes for classification. Finally, compare the results of the classification analysis, the classification of the most suitable type and classification of eigenvalues best in the classifier.
\end{abstract}

Keywords-EEG; motor imagery; AR model; feature extraction and classification

\section{INTRODUCTION}

In our country, stroke brings a heavy burden to family and society. Brain-computer interface (BCI) technology converts the brain electrical signal to the corresponding user command, so as to realize the human brain and computer or other electronic devices between direct communication and control, it can provide someone who thinking normal but with movement disorder a way to communicate with the outside world and an effective way to control. Imaginary motion EEG belongs to imagine EEG signals, it does not need to external stimuli, brain imagine some movement only can produce the related characteristics of EEG signals, it is a a practical and feasible method of brain-computer interface study. Therefore this article will choose imaginary motion EEG as the research object.

The development of brain-computer interface technology is closely related to brain electrical signal processing technology, It will promote the progress of BCI technology to a great extent by improving the EEG processing technology, which can improve the identification and classification accuracy of imaginary Motion EEG .

At present, the imaginary motion EEG research is still at the laboratory stage, the space is large, and BCI technology research is of great significance. In pretreatment, we commonly used bandpass filter and a trap filter the collected EEG, besides, there are some related denoising algorithm, for example, independent component analysis (ICA), principal component analysis (PCA) and wavelet transform, etc. In terms of feature extraction, there are methods include common space model, wavelet analysis, and the autoregressive (AR) model, etc.

\section{EMPIRICAL DATA}

The EEG data in this paper are adopted from the BCI2005 international competition, they are. Mat format, 60 channels, the left mastoid as the reference electrode, right mastoid is grounded. C3, Cz, C4 channels are corresponded to electrode number 28, 31, and 34. Sampling frequency is $250 \mathrm{~Hz}$, filtering frequency is $1-50$ $\mathrm{Hz}$, then sending the data into $50 \mathrm{~Hz}$ notch filter. It is $7 \mathrm{~s}$ to complete a single test, a test's full time is $10.24 \mathrm{~s}$ with break time, there are 2560 samples in total. The specific acquisition scheme is as follows:

1) the subjects sat quietly in the display waiting for the start of the experiment, the time is $2 \mathrm{~s}$, during which the screen shows "+"; 
2) when $t=2 \mathrm{~s}$, the screen shows "+";

3) when $t=3$, arrows last for $1 \mathrm{~s}$, during which the participants begin to imagine, until the end of 7 seconds.

Left hand, right hand, tongue, foot imagine in random order, each of them shows ten times. They are shown in figure 1 below:

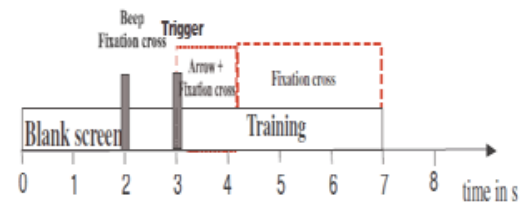

Fig. 1. The process of a single imagine movement test.

\section{DATA ANALYSIS}

A. The pretreatment of the EEG signals

In this article, we used cycle diagram method, the autocorrelation method and method of autoregressive (AR) model to analysis the single data of the original EEG power spectrum before pretreatment. The 140th test corresponds to the left hand movement, we choose the test data, respectively, using three kinds of power spectrum analysis method to draw the C3 and C4 channel power spectrum curve.

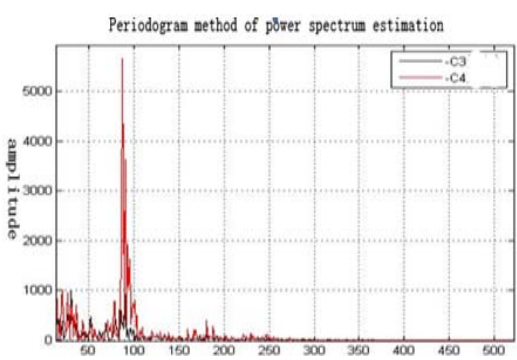

Fig. 2. Periodogram method of power spectrum estimation.

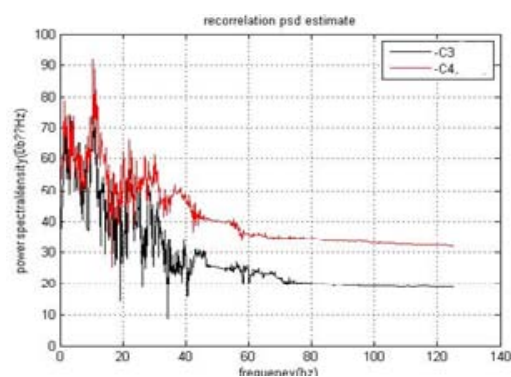

Fig. 3. Autocorrelation method of power spectrum estimation.

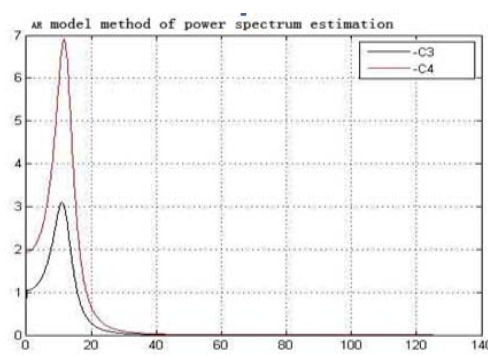

Fig. 4. AR model power spectrum estimation.

Through the analysis of power spectrum diagram: at about $10 \mathrm{~Hz}$ frequency power, spectrum amplitude of C3 channel was obviously higher than that of $\mathrm{C} 4$ channel, 8 and $13 \mathrm{~Hz}$ frequency contains the characteristics of imaginary motion EEG. So extracting the EEG from frequency range should include at least 8 to $13 \mathrm{~Hz}$ frequency band.

In this paper, using the ICA algorithm for signal denoising, first of all, according to the principle of the algorithm, choose mixed signals unmixed, they are the brain electrical signal and noise signal, then using ICA get EEG and noise, after it, we can take out not only the correlation, but also meet the mutual statistical independence.

Below it's the analysis of ERD/ERS of time domain of left hand movement imaginary :

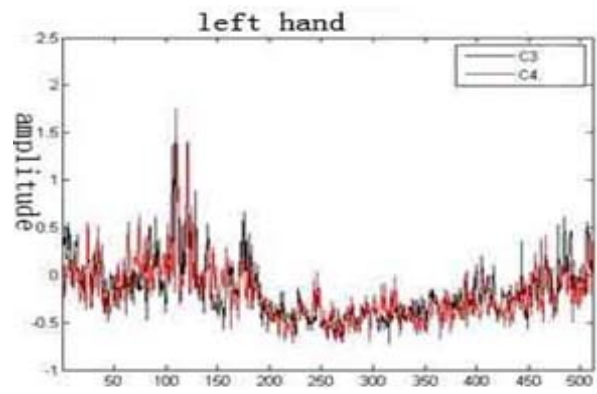

Fig. 5. Left hand ERD/ERS analysis of time domain.

We can see from the picture, when the movement imagination comes from left hand, ERD curve of C3 channel was obviously higher than that of C4 channels, while the right hand is opposite. Find corresponding points which distinguish clearly the differences between the C3 and C4 channels, so as to intercept the segment preprocessed of the time domain signal for the feature extraction for next step.

\section{B. Feature extraction}

This article selects three feature extraction methods for feature extraction in time domain and frequency domain respectively: setting AR model, extracting EEG features from time domain. By observing the frequency spectrum analysis and time domain ERD analysis to extract 
specific frequency and time signal, and looking on this signal energy as the characteristic value, extracting feature from time and frequency domain by wavelet analysis.

AR model's idea is to use the establishment of regression model approximately painting real EEG signals, the main of setting up AR model is to determine the model order, determine the order number includes the variance, the rule of FPE, AIC criterion and BIC rule. After getting the order number of the model, using the burg algorithm calculation model coefficient, in order to implement the statement, we use $[\mathrm{a}, \mathrm{e}]=$ arburg (x, p) in matlab, in which a is the model of the coefficient matrix, e is the variance of noise $u(n), x$ is EEG data of model, $p$ is the model order of the above guideline.

We can intercept signal through bandpass filter in the time domain, the signal obviously obtains

features unsynchronized, an obvious change is the signal energy has larger changes, and the change of energy is closely related to the changes of signal amplitude, at the same time. So from the frequency domain, we can intercept one of estimations of AR model power spectrum such as C3 and C4's channel power spectrum amplitude whose difference is bigger as a characteristic value that. From the time domain, we can be used the signal amplitude of the square as a characteristic value through filter and after being intercepted.

This paper, using wavelet packet decompose the signal into different frequency bands, then refacting the spectrum in the node contains useful information. The decomposition function in matlab is wpdec, it has 4 decomposition layers, the selected wavelet base is coif4, refactoring function is wprcoef.

\section{THE CLASSIFICATION OF CHARACTERISTICS}

\section{A. Classifier based on BP neural network}

The design principle of the BP neural network classifier is based on artificial neuron, when use it, we must determine the layers and the number of neurons in the network .The classification is multilayer feedforward network according to the error backward propagation algorithm, including the input layer, hidden layer and output layer, when use ,we can see the input layer and output layer, we can input training and testing data from the input layer , output layer output the category of the matrix compare to the known category, from which we can decide the training effect and the validity of the characteristic value of the network. BP classifier adjust network weights and threshold of constantly by back propagation so that the sum of the error squares of the network is the smallest.

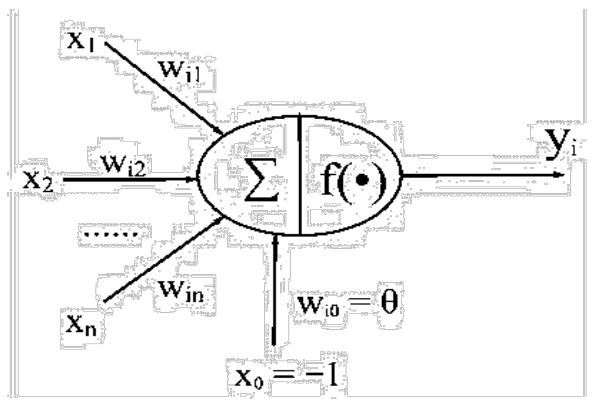

Fig. 6. artificial neural network model.

Implementation of the proposed algorithm includes three phases: creating a network, this article uses the newff function; The second is the training of neural network, using the train function, when training, we input the characteristic matrix of training data and test corresponding category matrix; The third is calculation of the classification accuracy of the test data through trained network by sim function.

The classifier's accuracy will be changed according to AR model's oeder during the feature extraction stage.Table 1 is using the BP neural network classifier to train the characteristic value of different types and the classification accuracy of testing.

TABLE1. THE BP NEURAL NETWORK CLASSIFICATION

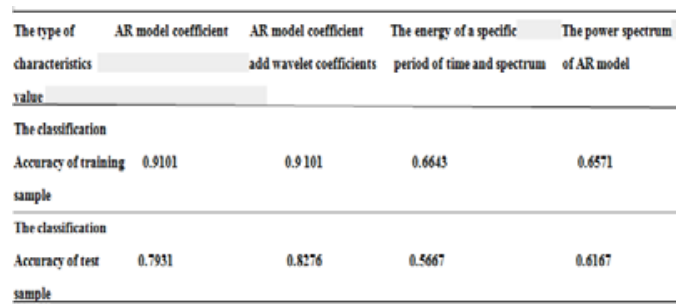

Data in the table have been trained many times. When add AR model coefficient and wavelet coefficients's average energy as a characteristic value, classification accuracy at about 0.9 after each training, but only look on AR model coefficient as characteristic value, classification results were often less than the former.

B. Classifier based on probabilistic neural network (PNN)

PNN neural network classifier is probabilistic neural network classifier, which is based on the probability density function of statistics for the knowledge related to parameter estimation, there is no feedback of the forward propagation algorithm. The network consists of input layer, samples, summation layer and output layer, the corresponding number of neurons in 
each layer are the dimensions of the feature vector, the training sample number, number of categories and 1.One of the sample layer activation function is Gaussian function.

Compared with the BP neural network, the PNN neural network is faster, classification accuracy is higher in addition, the error tolerance is better. Deficiency is that it is the requirement of the training sample's representative is higher, which we often say characteristic matrix, storage space is big. When to create network we use new pnn function in matlab firstly, and then test by sim function. Classification results are shown in table 2.

TABLE2. CLASSIFICATION OF PROBABILITY NEURAL

NETWORK

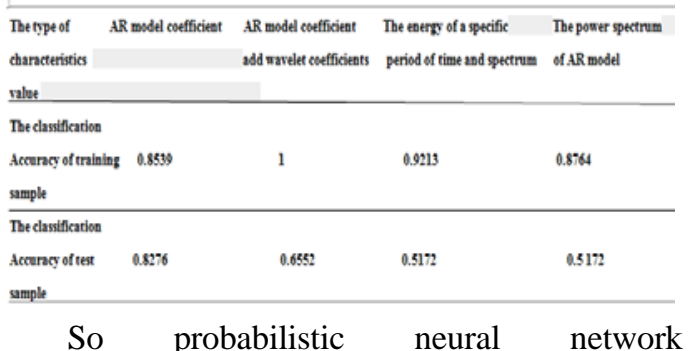
classification is the best dealing with all kinds of characteristic value, especially in characterized by AR model add wavelet coefficient, value accuracy is 1 .

\section{CONCLUSION}

For AR model coefficient, the AR model coefficient add wavelet coefficient of a specific time period, spectrum signal energy, AR model power spectrum these four eigenvalues, for the AR model coefficients and AR model coefficient add wavelet coefficient's average energy, two classification methods are ideal. Specific time period, the energy spectrum and AR model power spectrum of these two kinds of characteristic value in the classification of these two kinds of classifier's accuracy is higher. From these two kinds of classifier classification's effect, if the test data is the training data, the classification accuracy is higher, especially for the first two kinds of characteristic value, the classification accuracy is above $85 \%$; If the test data is different from the training data, accuracy will be low. PNN neural network's classification accuracy is higher when data from testing samples, it shows that the classification method appeared over fitting phenomenon. From the characteristics of various values of the differences between the training sample and test sample's classification accuracy, the BP neural network is relatively stable.
So, if we work on imaginary motion EEG extraction and classification processing, we can choose the AR model and AR model combined with wavelet coefficients as feature vector, choose BP neural network as classification method.

\section{REFERENCE}

[1] Wang Yichen. Prevention on stroke.Shanghai insurance, 2008 (6) : 22 to 23

[2] Cheng Peiqing,Digital signal processing[M],Tsinghua university press,2011:343

[3] Hei Meng,Zhang Xiuxiu. Progress of feature extraction and classification of imagine eeg [J]. Journal biomedical engineering in Beijing, 2013, 32 (2) : 2 to 3

[4] Wu Xiaopei, Independent component analysis and its application in the brain electrical signal processing, China university of science and technology [D], 2002, 11 to 12

[5] Xi Jia, research on brain-machine interface of brain electrical signal's feature extraction and classification [D], hebei university of technology, 2007:17to18

[6] Fengying Wang Li-li Chang. Power spectrum estimation and matlab simulation [J], shandong university of science and technology, 2006:1to2

[7] Zou Qing, EEG feature extraction and recognition based on AR model [D], southeast university, 2009:15 